\title{
IMWG Sustained MRD-negative
}

National Cancer Institute

\section{Source}

National Cancer Institute. IMWG Sustained MRD-negative. NCI Thesaurus. Code C159800.

Minimal residual disease negativity in the marrow, by next-generation flow cytometry or next-generation sequencing, or both, and by imaging, confirmed a minimum of 1 year apart. 